\title{
Association between Serum Dipeptidyl Peptidase-4 Concentration and Obesity-Related Factors in Health Screen Examinees (J Obes Metab Syndr 2017;26:188-96)
}

\author{
Ji Yeon Lee', Byoung Kuk Jang 1,2, Min Kyung Song ${ }^{3}$, Hye Soon Kim¹, Mi-Kyung Kim ${ }^{1, *}$ \\ 'Department of Internal Medicine, Keimyung University School of Medicine, Daegu; ${ }^{2}$ Institute for Cancer Research, \\ Keimyung University, Daegu: ${ }^{3}$ Department of Food Science and Nutrition, Graduate School, Keimyung University, \\ Daegu, Korea
}

\author{
Received February 13, 2018 \\ Reviewed February 22, 2018 \\ Accepted February 25, 2018 \\ ${ }^{*}$ Corresponding author \\ Mi-Kyung Kim \\ https://orcid.org/0000-0001-5750-3598 \\ Department of Internal Medicine, \\ Keimyung University School of Medicine, \\ 56 Dalseong-ro, Jung-gu, Daegu 41931, \\ Korea \\ Tel: +82-53-250-7486 \\ Fax: +82-53-250-7982 \\ E-mail:mdkmk@dsmc.or.kr
}

Dipeptidyl peptidase-4 (DPP-4), a serine protease, degrades peptides containing alanine or proline residues and amino-terminal residues of proteins. ${ }^{1}$ It is an aminopeptidase found in most tissues of the body, including the liver, lung, kidney, intestine, lymph nodes, and endothelial cells, and resides on cell membranes, where it exerts its effect. Physiological effects of DPP-4 can be broadly divided into enzymatic effects such as the inhibition of incretins and non-enzymatic effects associated with immune regulation, behavioral response, and inflammation depending on the substance on which DPP-4 acts. ${ }^{2,3}$ Since Lamers et al. ${ }^{4}$ reported DPP-4 as novel adipokine, many studies have shown serum DPP-4 level or activity to be associated with obesity, diabetes mellitus, and fatty liver. ${ }^{5,6}$ Our previous study showed serum DPP-4 concentration was positively correlated with lean body mass, total cholesterol, and creatinine and was elevated in the obese group compared to the normal weight group, as reported in Journal of Obesity \& Metabolic Syndrome. ${ }^{7}$ It is an honor to reply to a Letter to the Editor with great comments on our study. Thus, we kindly respond to the issues raised in the Letter.

Our studies evaluated whether serum DPP-4 concentration is associated with obesity and obesity-related factors such as glucose, lipid profile, and body fat using blood test and bio-impedance analysis. Even though our study showed that serum DPP-4 level was higher in the obese group, these studies indirectly reflect the relationship between DPP-4 and obesity. Therefore, there is a limitation to understanding the mechanism and role of DPP-4 in adipose tissue. Recently, some studies have supported the association between DPP-4 and obesity. Lamer et al. ${ }^{4}$ showed higher release of DPP-4 in fully differentiated adipocytes than in preadipocytes, and that direct addition of DPP-4 to fat and skeletal muscles impaired insulin signaling, suggesting that DPP-4 is an adipokine and might have direct effects on adipose tissue. To assess the tissue sources of circulating DPP-4, Sell et al. ${ }^{8}$ assessed DPP-4 expression and release in the adipose tissue of lean and obese patients. Their results showed that DPP-4 expression was correlated with body mass index and was higher in visceral adipose tissue (VAT) than subcutaneous adipose tissue (SAT). It was also increased in VAT of lean patients with impaired glucose tolerance. In addition, DPP-4 release is higher in VAT than in SAT in both lean and obese patients. Moreover, Stengel et al. ${ }^{9}$ 
showed that DPP-4 protein level in the plasma was higher in obese patients and correlated with body mass index. These studies suggest that adipose tissue could be a primary source of higher serum DPP-4 or activity. However, the exact mechanisms of DPP-4 on adipose tissue are not known yet. Therefore, further studies are needed to elucidate the functional role of DPP-4 and its contribution to the incretin system. Finally, we thank you for the Letter and the opportunity to respond. We hope that Journal of Obesity \& Metabolic Syndrome will continue to prosper in the future.

\section{CONFLICTS OF INTEREST}

The authors declare no conflict of interest.

\section{REFERENCES}

1. Mentlein R. Dipeptidyl-peptidase IV (CD26): role in the inactivation of regulatory peptides. Regul Pept 1999;85:9-24.

2. Drucker DJ, Nauck MA. The incretin system: glucagon-like peptide- 1 receptor agonists and dipeptidyl peptidase- 4 inhibitors in type 2 diabetes. Lancet 2006;368:1696-705.

3. Zhong J, Rao X, Rajagopalan S. An emerging role of dipeptidyl peptidase 4 (DPP4) beyond glucose control: potential implications in cardiovascular disease. Atherosclerosis 2013;226: 305-14.
4. Lamers D, Famulla S, Wronkowitz N, Hartwig S, Lehr S, Ouwens DM, et al. Dipeptidyl peptidase 4 is a novel adipokine potentially linking obesity to the metabolic syndrome. Diabetes 2011;60:1917-25.

5. Williams KH, Vieira De Ribeiro AJ, Prakoso E, Veillard AS, Shackel NA, Brooks B, et al. Circulating dipeptidyl peptidase-4 activity correlates with measures of hepatocyte apoptosis and fibrosis in non-alcoholic fatty liver disease in type 2 diabetes mellitus and obesity: a dual cohort cross-sectional study. J Diabetes 2015;7:809-19.

6. Kirino Y, Sei M, Kawazoe K, Minakuchi K, Sato Y. Plasma dipeptidyl peptidase 4 activity correlates with body mass index and the plasma adiponectin concentration in healthy young people. Endocr J 2012;59:949-53.

7. Lee JY, Jang BK, Song MK, Kim HS, Kim MK. Association between serum dipeptidyl peptidase- 4 concentration and obesity-related factors in health screen examinees. J Obes Metab Syndr 2017;26:188-96.

8. Sell H, Blüher M, Klöting N, Schlich R, Willems M, Ruppe F, et al. Adipose dipeptidyl peptidase-4 and obesity: correlation with insulin resistance and depot-specific release from adipose tissue in vivo and in vitro. Diabetes Care 2013;36:4083-90.

9. Stengel A, Goebel-Stengel M, Teuffel P, Hofmann T, Buße P, Kobelt $\mathrm{P}$, et al. Obese patients have higher circulating protein levels of dipeptidyl peptidase IV. Peptides 2014;61:75-82. 